\title{
Oscillation criteria for nonlinear functional differential equations of neutral type
}

\section{Fatima N Ahmed*, Rokiah Rozita Ahmad, Ummul Khair Salma Din and Mohd Salmi Md Noorani}

\section{"Correspondence:}

zahra80zahra@yahoo.com

School of Mathematical Sciences,

Faculty of Science and Technology,

Universiti Kebangsaan Malaysia,

43600 UKM, Bangi, Selangor D.E.,

Malaysia

\begin{abstract}
In this paper, some new sufficient conditions for the oscillation of all solutions of nonlinear neutral delay differential equations are established aiming at extending and/or improving some well known results in the literature. Our main results are obtained by employing the Riccati transformation aiming to transfer the neutral equation to a nonneutral type and then using some inequality techniques. Some illustrative examples are also included.
\end{abstract}

MSC: $54 \mathrm{H} 25 ; 54 \mathrm{C} 60 ; 54 \mathrm{E} 50$

Keywords: oscillations; neutral delay differential equations; nonlinear

\section{Introduction}

When delays appear in additional terms involving the highest order derivative of the unknown function in a differential equation, we are dealing with a neutral type differential equation. The study of the asymptotic and oscillatory behavior of solutions of neutral differential equations is of importance in applications. This is due to the fact that such equations appear in various phenomena including networks containing lossless transmission lines (as in high-speed computers where such lines are used to interconnect switching circuits), in the study of vibrating masses attached to an elastic bar, as the Euler equations for the minimization of functionals involving a time delay in some variational problems and in the theory of automatic control (see Hale [1], Driver [2] and Boe and Chang [3] and references cited therein). The construction of these models using delays is complemented by the mathematical investigation of nonlinear equations.

In this paper, we mainly consider the nonlinear neutral delay differential equations of the form

$$
[r(t)(a(t) x(t)+p(t) x(t-\tau))]^{\prime}+q(t) f(x(t-\sigma))=0,
$$

where

$$
r(t), a(t), p(t), q(t) \in C\left[\left[t_{0}, \infty\right), \mathbb{R}^{+}\right], \quad \tau, \sigma \in \mathbb{R}^{+},
$$

and $f$ satisfies

$$
f \in C[\mathbb{R}, \mathbb{R}], \quad u f(u)>0 \quad \text { for } u \neq 0,
$$

\section{Springer}

(c) 2015 Ahmed et al.; licensee Springer. This is an Open Access article distributed under the terms of the Creative Commons Attribution License (http://creativecommons.org/licenses/by/4.0), which permits unrestricted use, distribution, and reproduction in any medium, provided the original work is properly credited. 
and there exists a positive constant $k_{0}$ such that

$$
\frac{f(u)}{u} \geq k_{0}>0 .
$$

Many authors have considered linear neutral delay differential equations and established sufficient conditions for oscillation of all solutions. We refer to Ahmed et al. [4], Karpuz and Ocalan [5], Gopalsamy et al. [6] and Zhou [7].

In 2002, Saker and Kubiaczyk [8] considered the nonlinear neutral equation

$$
(a(t) x(t)+p(t) x(t-\tau))^{\prime}+q(t) f(x(t-\sigma))=0,
$$

where the function $f$ satisfies condition (1.3) with $\lim _{u \rightarrow 0} \frac{f(u)}{u}=k_{1}$ exists and there exists $t^{*} \geq t_{0}$ such that

$$
\frac{p\left(t^{*}+n^{*} \tau\right)}{a\left(t^{*}+\left(n^{*}-1\right) \tau\right)} \leq 1 \quad \text { for } n^{*}=0,1,2, \ldots
$$

In 2003, Kubiaczyk et al. [9] studied the nonlinear equations of the form

$$
(x(t)+p(t) x(t-\tau))^{\prime}+q(t) \prod_{i=1}^{n}\left|x\left(t-\sigma_{i}\right)\right|^{\alpha_{i}} \operatorname{sign} x\left(t-\sigma_{i}\right)=0,
$$

where $\alpha_{i}>0$ and $\sum_{i=1}^{n} \alpha_{i}=1$, and they have given some sharp sufficient conditions for the oscillation of all solutions of (1.7).

In 2004, Graef et al. [10] considered (1.1) when $a(t) \equiv 1, r(t) \equiv 1$ and developed some sufficient conditions for the oscillation of all solutions. For further results on the oscillation of various classes of neutral differential equations one can see [11-17].

A primary purpose of this paper is to establish new integral conditions that guarantee the oscillation of all solutions of $(1.1)$ when $p(t)$ is constant and equal $p_{0}$. In some sense, the obtained results here extend and generalize several of well known results in the literature.

Let $m=\max \{\tau, \sigma\}$. By a solution of (1.1) we mean a function $x \in C\left[\left[t_{1}-m, \infty\right), \mathbb{R}\right]$ for some $t_{1} \geq t_{0}$ such that $a(t) x(t)+p(t) x(t-\tau)$ is continuously differentiable for $t \geq t_{1}$ and such that (1.1) is satisfied for $t \geq t_{1}$.

Let $t_{1} \geq t_{0}$ be a given initial point and let $\Phi \in C\left[\left[t_{1}-m, t_{1}\right], \mathbb{R}\right]$ be a given initial function. Then one can show by using the method of steps that (1.1) has a unique solution on $\left[t_{1}, \infty\right)$ satisfying the initial function

$$
x(t)=\Phi(t), \quad \text { for } t_{1}-m \leq t \leq t_{1} .
$$

As is customary, a solution of (1.1) is said to be oscillatory if it has arbitrarily large zeros. Otherwise the solution is said to be nonoscillatory. Equation (1.1) is said to be oscillatory if all its solutions are oscillatory.

In the sequel, unless otherwise specified, when we write a functional inequality we shall assume that it holds for all sufficiently large values of $t$. 


\section{Auxiliary lemmas}

We give here some useful lemmas which will play an important role in the study of the oscillation of (1.1).

Lemma 2.1 [18] Assume that $P_{i}(t), \tau_{i}(t) \in C\left[\left[t_{0}, \infty\right),[0, \infty)\right], i=1,2, \ldots$.

Then the differential inequality

$$
x^{\prime}(t)+\sum_{i=1}^{n} P_{i}(t) x\left(t-\tau_{i}(t)\right) \leq 0
$$

has an eventually positive solution if and only if the equation

$$
x^{\prime}(t)+\sum_{i=1}^{n} P_{i}(t) x\left(t-\tau_{i}(t)\right)=0
$$

has an eventually positive solution.

Lemma 2.2 [19] Assume that

$$
\limsup _{t \rightarrow \infty} \int_{t}^{t+\tau_{i}} p_{i}(s) d s>0, \quad \text { for some } i .
$$

If $x(t)$ is an eventually positive solution of the delay differential equation

$$
x^{\prime}(t)+\sum_{i=1}^{n} p_{i}(t) x\left(t-\tau_{i}\right)=0,
$$

then, for the same $i$,

$$
\liminf _{t \rightarrow \infty} \frac{x\left(t-\tau_{i}\right)}{x(t)}<\infty
$$

Lemma 2.3 Assume that

$$
\begin{aligned}
& a(t) \leq 1, \quad \sigma>\tau, \quad \text { and } \quad p_{0}>1, \\
& \limsup _{t \rightarrow \infty} \int_{t}^{t+\sigma-\tau} q(s) d s>0 .
\end{aligned}
$$

Let $x(t)$ be an eventually positive solution of the equation

$$
\left(a(t) x(t)+p_{0} x(t-\tau)\right)^{\prime}+q(t) f(x(t-\sigma))=0 .
$$

Set

$$
z(t)=a(t) x(t)+p_{0} x(t-\tau)
$$

then

$$
\liminf _{t \rightarrow \infty} \frac{z(t-\sigma+\tau)}{z(t)}<\infty .
$$


Proof In view of (2.7) and our hypothesis, we see that $z(t)>0$ eventually. From (2.8) we have

$$
z(t+\tau)=a(t+\tau) x(t+\tau)+p_{0} x(t)
$$

and

$$
p_{0} x(t-\tau)=z(t)-a(t) x(t) \geq z(t)-x(t) .
$$

Since $z^{\prime}(t)<0$, we have $z(t)>z(t+\tau)$, which implies with (2.10) that

$$
z(t) \geq p_{0} x(t) \Rightarrow x(t) \leq \frac{z(t)}{p_{0}} .
$$

Substituting in (2.11), we obtain

$$
p_{0} x(t-\tau) \geq z(t)-\frac{z(t)}{p_{0}}
$$

or

$$
p_{0}^{2} x(t-\tau) \geq p_{0} z(t)-z(t)
$$

Hence

$$
x(t-\sigma) \geq \frac{p_{0}-1}{p_{0}^{2}} z(t+\tau-\sigma) .
$$

From (2.7) and (2.12), we get

$$
z^{\prime}(t)+\frac{k_{0}\left(p_{0}-1\right)}{p_{0}^{2}} q(t) z(t+\tau-\sigma) \leq 0 .
$$

By Lemma 2.1, we find that

$$
z^{\prime}(t)+\frac{k_{0}\left(p_{0}-1\right)}{p_{0}^{2}} q(t) z(t+\tau-\sigma)=0
$$

has an eventually positive solution as well.

As a result, by Lemma 2.2 and (2.6), we have

$$
\liminf _{t \rightarrow \infty} \frac{z(t-\sigma+\tau)}{z(t)}<\infty
$$

which is the desired result. The proof is complete.

Lemma 2.4 Assume that conditions (2.5) hold. If (2.7) has an eventually positive solution, then

$$
\int_{t}^{t+\sigma-\tau} q(s) d s \leq \frac{p_{0}^{2}}{k_{0}\left(p_{0}-1\right)}
$$

for all sufficiently large $t$. 
Proof Proceeding as in the proof of Lemma 2.3, we again obtain the inequality (2.13). Integrating (2.13) from $t$ to $t+\sigma-\tau$, it follows that

$$
z(t-\tau+\sigma)-z(t)+\frac{k_{0}\left(p_{0}-1\right)}{p_{0}^{2}} \int_{t}^{t+\sigma-\tau} q(s) z(s-\sigma+\tau) d s \leq 0 .
$$

Using Bonnet's theorem, it follows that

$$
z(t-\tau+\sigma)-z(t)+\frac{k_{0}\left(p_{0}-1\right)}{p_{0}^{2}} z(t) \int_{t}^{t+\sigma-\tau} q(s) d s \leq 0 .
$$

Then

$$
z(t-\tau+\sigma)+\left(\frac{k_{0}\left(p_{0}-1\right)}{p_{0}^{2}} \int_{t}^{t+\sigma-\tau} q(s) d s-1\right) z(t) \leq 0 .
$$

Since $z(t)>0,(2.16)$ implies

$$
\frac{k_{0}\left(p_{0}-1\right)}{p_{0}^{2}} \int_{t}^{t+\sigma-\tau} q(s) d s-1 \leq 0 .
$$

Hence, for all sufficiently large $t$, we have

$$
\int_{t}^{t+\sigma-\tau} q(s) d s \leq \frac{p_{0}^{2}}{k_{0}\left(p_{0}-1\right)}
$$

which is the desired result. The proof is complete.

Remark 2.5 Lemma 2.3 and Lemma 2.4 extend results of Graef et al. [10].

\section{Oscillation of solutions}

Theorem 3.1 Assume that conditions (2.5) and (2.6) hold. If

$$
\int_{t_{0}}^{\infty} q(t) \ln \left(\frac{e k_{0}\left(p_{0}-1\right)}{p_{0}^{2}} \int_{t}^{t+\sigma-\tau} q(s) d s\right) d t=\infty
$$

Then every solution of (2.7) oscillates.

Proof For the sake of obtaining a contradiction, assume that there is an eventually positive solution $x(t)$ of (2.7). Set $z(t)$ as in (2.8). Then $z(t)$ is eventually positive and decreasing and satisfies the inequality (2.13). That is,

$$
z^{\prime}(t)+\frac{k_{0}\left(p_{0}-1\right)}{p_{0}^{2}} q(t) z(t+\tau-\sigma) \leq 0
$$

Let

$$
\psi(t)=-\frac{z^{\prime}(t)}{z(t)}
$$


Then $\psi(t)$ is continuous and nonnegative. So, there exists $t_{1} \geq t_{0}$ with $z\left(t_{1}\right)>0$ such that

$$
z(t)=z\left(t_{1}\right) \exp \left(-\int_{t_{1}}^{t} \psi(s) d s\right)
$$

Moreover, $\psi(t)$ satisfies

$$
\psi(t) \geq \frac{k_{0}\left(p_{0}-1\right)}{p_{0}^{2}} q(t) \exp \left(\int_{t+\tau-\sigma}^{t} \psi(s) d s\right)
$$

By using the inequality (cf. Erbe et al. [20], p.32)

$$
e^{r x} \geq x+\frac{\ln (e r)}{r}, \quad x, r>0
$$

we have from (3.2)

$$
\begin{aligned}
\psi(t) & \geq \frac{k_{0}\left(p_{0}-1\right)}{p_{0}^{2}} q(t) \exp \left(\frac{A(t)}{A(t)} \int_{t+\tau-\sigma}^{t} \psi(s) d s\right) \\
& \geq \frac{k_{0}\left(p_{0}-1\right)}{p_{0}^{2}} q(t) \exp \left(\frac{1}{A(t)} \int_{t+\tau-\sigma}^{t} \psi(s) d s+\frac{\ln (e A(t))}{A(t)}\right),
\end{aligned}
$$

where

$$
A(t)=\frac{k_{0}\left(p_{0}-1\right)}{p_{0}^{2}} \int_{t}^{t+\sigma-\tau} q(s) d s
$$

Therefore,

$$
\psi(t) \int_{t}^{t+\sigma-\tau} q(s) d s-q(t) \int_{t+\tau-\sigma}^{t} \psi(s) d s \geq q(t) \ln \left(\frac{e k_{0}\left(p_{0}-1\right)}{p_{0}^{2}} \int_{t}^{t+\sigma-\tau} q(s) d s\right) .
$$

Hence, for $\xi>T+\sigma-\tau$,

$$
\begin{gathered}
\int_{T}^{\xi} \psi(t)\left(\int_{t}^{t+\sigma-\tau} q(s) d s\right) d t-\int_{T}^{\xi} q(t)\left(\int_{t+\tau-\sigma}^{t} \psi(s) d s\right) d t \\
\geq \int_{T}^{\xi} q(t) \ln \left(\frac{e k_{0}\left(p_{0}-1\right)}{p_{0}^{2}} \int_{t}^{t+\sigma-\tau} q(s) d s\right) d t .
\end{gathered}
$$

By interchanging the order of integration, we have

$$
\int_{T}^{\xi} q(t)\left(\int_{t+\tau-\sigma}^{t} \psi(s) d s\right) d t \geq \int_{T}^{\xi+\tau-\sigma} \psi(t)\left(\int_{t}^{t+\sigma-\tau} q(s) d s\right) d t
$$

Combining (3.3) and (3.4), leads to

$$
\int_{\xi+\tau-\sigma}^{\xi} \psi(t)\left(\int_{t}^{t+\tau-\sigma} q(s) d s\right) d t \geq \int_{T}^{\xi} q(t) \ln \left(\frac{e k_{0}\left(p_{0}-1\right)}{p_{0}^{2}} \int_{t}^{t+\sigma-\tau} q(s) d s\right) d t
$$

Using (2.15) of Lemma 2.4 in (3.5), we obtain

$$
\int_{\xi+\tau-\sigma}^{\xi} \psi(t) d t \geq \frac{k_{0}\left(p_{0}-1\right)}{p_{0}^{2}} \int_{T}^{\xi} q(t) \ln \left(\frac{e k_{0}\left(p_{0}-1\right)}{p_{0}^{2}} \int_{t}^{t+\sigma-\tau} q(s) d s\right) d t
$$


i.e.,

$$
\ln \frac{z(\xi+\tau-\sigma)}{z(\xi)} \geq \frac{k_{0}\left(p_{0}-1\right)}{p_{0}^{2}} \int_{T}^{\xi} q(t) \ln \left(\frac{e k_{0}\left(p_{0}-1\right)}{p_{0}^{2}} \int_{t}^{t+\sigma-\tau} q(s) d s\right) d t .
$$

This result along with condition (3.1) leads to

$$
\lim _{t \rightarrow \infty} \frac{z(t+\tau-\sigma)}{z(t)}=\infty
$$

which contradicts (2.9) and completes the proof.

Example 3.2 Consider the equation

$$
\left(\frac{1}{t} x(t)+3 x(t-1)\right)^{\prime}+\frac{9}{2 e}\left(1+\frac{1}{t}\right) x(t-2)\left(1+x^{2}(t-2)\right)=0, \quad t \geq e .
$$

Here we have

$$
\begin{aligned}
& a(t)=\frac{1}{t}, \quad q(t)=\frac{9}{2 e}\left(1+\frac{1}{t}\right), \quad p_{0}=3, \quad \tau=1, \quad \text { and } \quad \sigma=2, \\
& f(x(t-\sigma))=x(t-2)\left(1+x^{2}(t-2)\right) .
\end{aligned}
$$

Then we have

$$
\left.\frac{f(u)}{u}=\frac{x(t-2)\left(1+x^{2}(t-2)\right)}{x(t-2)}=1+x^{2}(t-2)\right) \geq 1 .
$$

Let $k_{0}=1$,

$$
\begin{aligned}
& \int_{t_{0}}^{\infty} q(t) \ln \left(\frac{e k_{0}\left(p_{0}-1\right)}{p_{0}^{2}} \int_{t}^{t+\sigma-\tau} q(s) d s\right) d t \\
& \quad=\int_{e}^{\infty} \frac{9}{2 e}\left(1+\frac{1}{t}\right) \ln \left(\int_{t+1}^{t+2}\left(1+\frac{1}{s}\right) d s\right) d t \\
& \quad=\frac{9}{2 e} \int_{e}^{\infty}\left(1+\frac{1}{t}\right)\left(1+\ln \left(1+\frac{1}{t}\right)\right) \\
& \quad \geq \frac{9}{2 e} \int_{e}^{\infty} \ln \left(1+\ln \left(1+\frac{1}{t}\right)\right)=\infty .
\end{aligned}
$$

Hence, according to Theorem 3.1, all solutions of (3.6) oscillate.

Remark 3.3 Theorem 3.1 extends results of Graef et al. ([10], Theorem 2.1 and Theorem 2.2), where $a(t) \equiv 1$.

Theorem 3.4 Assume that conditions (2.5) hold. If

$$
0<c \leq \liminf _{t \rightarrow \infty} \int_{t}^{t-\tau+\sigma} \frac{q(s)}{r(s+\tau-\sigma)} d s
$$


and

$$
\int_{t_{0}}^{\infty} \frac{q(t)}{r(t+\tau-\sigma)} \ln \left(\frac{e k_{0}\left(p_{0}-1\right)}{p_{0}^{2}} \int_{t}^{t+\sigma-\tau} \frac{q(s)}{r(s+\tau-\sigma)} d s\right) d t=\infty .
$$

Then every solution of (1.1) oscillates.

Proof For the sake of obtaining a contradiction, assume that there is an eventually positive solution $x(t)$ of (1.1). Set $z(t)$ as in (2.8). Then $z(t)$ is eventually positive and decreasing. From (2.12) and (1.1), we have

$$
(r(t) z(t))^{\prime}+\frac{k_{0}\left(p_{0}-1\right)}{p_{0}^{2}} q(t) z(+\tau-\sigma) \leq 0 .
$$

Dividing (3.9) by $r(t)>0$, we obtain

$$
z^{\prime}(t)+\frac{r^{\prime}(t)}{r(t)} z(t)+\frac{k_{0}\left(p_{0}-1\right)}{p_{0}^{2}} \frac{q(t)}{r(t)} z(+\tau-\sigma) \leq 0 .
$$

Let

$$
z(t)=\exp \left(-\int_{t_{0}}^{t} \frac{r^{\prime}(s)}{r(s)} d s\right) y(t)
$$

which implies that $y(t)>0$. Substituting in (3.10) yields for all $t \geq t_{0}$,

$$
y^{\prime}(t)+\frac{k_{0}\left(p_{0}-1\right)}{p_{0}^{2}} \frac{q(t)}{r(t+\tau-\sigma)} y(+\tau-\sigma) \leq 0 .
$$

Set

$$
\lambda(t)=-\frac{y^{\prime}(t)}{y(t)}
$$

Then $\lambda(t)$ is continuous and positive. So, there exists $t_{1} \geq t_{0}$ with $y\left(t_{1}\right)>0$ such that

$$
y(t)=y\left(t_{1}\right) \exp \left(\int_{t_{1}}^{t} \lambda(s) d s\right) .
$$

Moreover, $\lambda(t)$ satisfies

$$
\lambda(t) \geq \frac{k_{0}\left(p_{0}-1\right)}{p_{0}^{2}} \frac{q(t)}{r(t+\tau-\sigma)} \exp \left(\int_{t+\tau-\sigma}^{t} \lambda(s) d s\right) .
$$

Applying the inequality

$$
e^{r x} \geq x+\frac{\ln (e r)}{r}, \quad x, r>0
$$

to (3.13) yields that

$$
\begin{aligned}
\lambda(t) & \geq\left(\frac{k_{0}\left(p_{0}-1\right)}{p_{0}^{2}}\right)\left(\frac{q(t)}{r(t+\tau-\sigma)}\right) \exp \left(\frac{B(t)}{B(t)} \int_{t+\tau-\sigma}^{t} \lambda(s) d s\right) \\
& \geq\left(\frac{k_{0}\left(p_{0}-1\right)}{p_{0}^{2}}\right)\left(\frac{q(t)}{r(t+\tau-\sigma)}\right)\left(\frac{1}{B(t)} \int_{t+\tau-\sigma}^{t} \lambda(s) d s+\frac{\ln (e B(t))}{B(t)}\right),
\end{aligned}
$$


where

$$
B(t)=\frac{k_{0}\left(p_{0}-1\right)}{p_{0}^{2}} \int_{t}^{t-\tau+\sigma} \frac{q(s)}{r(s+\tau-\sigma)} d s
$$

Therefore,

$$
\begin{gathered}
\lambda(t) \int_{t}^{t-\tau+\sigma} \frac{q(s)}{r(s+\tau-\sigma)} d s-\frac{q(t)}{r(t+\tau-\sigma)} \int_{t+\tau-\sigma}^{t} \lambda(s) d s \\
\quad \geq \frac{q(t)}{r(t+\tau-\sigma)} \ln \left(\frac{e k_{0}\left(p_{0}-1\right)}{p_{0}^{2}} \int_{t}^{t-\tau+\sigma} \frac{q(s)}{r(s+\tau-\sigma)} d s\right) .
\end{gathered}
$$

Hence, for $\eta>T_{1}+\sigma-\tau$

$$
\begin{aligned}
& \int_{T_{1}}^{\eta} \lambda(t)\left(\int_{t}^{t-\tau+\sigma} \frac{q(s)}{r(s+\tau-\sigma)} d s\right) d t-\int_{T_{1}}^{\eta} \frac{q(t)}{r(t+\tau-\sigma)}\left(\int_{t+\tau-\sigma}^{t} \lambda(s) d s\right) d t \\
& \quad \geq \int_{T_{1}}^{\eta} \frac{q(t)}{r(t+\tau-\sigma)} \ln \left(\frac{e k_{0}\left(p_{0}-1\right)}{p_{0}^{2}} \int_{t}^{t-\tau+\sigma} \frac{q(s)}{r(s+\tau-\sigma)} d s\right) d t .
\end{aligned}
$$

By interchanging the order of integration, we have

$$
\int_{T_{1}}^{\eta} \frac{q(t)}{r(t+\tau-\sigma)}\left(\int_{t+\tau-\sigma}^{t} \lambda(s) d s\right) d t \geq \int_{T_{1}}^{\eta+\tau-\sigma} \lambda(t)\left(\int_{t}^{t-\tau+\sigma} \frac{q(s)}{r(s+\tau-\sigma)} d s\right) d t .
$$

From (3.14) and (3.15), we obtain

$$
\begin{aligned}
& \int_{\eta+\tau-\sigma}^{\eta} \lambda(t)\left(\int_{t}^{t-\tau+\sigma} \frac{q(s)}{r(s+\tau-\sigma)} d s\right) d t \\
& \quad \geq \int_{T_{1}}^{\eta} \frac{q(t)}{r(t+\tau-\sigma)} \ln \left(\frac{e k_{0}\left(p_{0}-1\right)}{p_{0}^{2}} \int_{t}^{t-\tau+\sigma} \frac{q(s)}{r(s+\tau-\sigma)} d s\right) d t .
\end{aligned}
$$

Employing (2.15) in (3.16), it follows that

$$
\int_{\eta+\tau-\sigma}^{\eta} \lambda(t) d t \geq \frac{k_{0}\left(p_{0}-1\right)}{p_{0}^{2}} \int_{T_{1}}^{\eta} \frac{q(t)}{r(t+\tau-\sigma)} \ln \left(\frac{e k_{0}\left(p_{0}-1\right)}{p_{0}^{2}} \int_{t}^{t-\tau+\sigma} \frac{q(s)}{r(s+\tau-\sigma)} d s\right) d t
$$

or

$$
\begin{aligned}
& \ln \frac{y(\eta+\tau-\sigma)}{y(\eta)} \\
& \quad \geq \frac{k_{0}\left(p_{0}-1\right)}{p_{0}^{2}} \int_{T_{1}}^{\eta} \frac{q(t)}{r(t+\tau-\sigma)} \ln \left(\frac{e k_{0}\left(p_{0}-1\right)}{p_{0}^{2}} \int_{t}^{t-\tau+\sigma} \frac{q(s)}{r(s+\tau-\sigma)} d s\right) d t .
\end{aligned}
$$

From (3.17) and (3.9), we have

$$
\lim _{t \rightarrow \infty} \frac{y(t+\tau-\sigma)}{y(t)}=\infty
$$


On the other hand, from condition (3.8), there exists a sequence $\left\{t_{n}\right\}, t_{n} \rightarrow \infty$ as $n \rightarrow \infty$, and there exists $\mu_{n} \in\left(t_{n}-\sigma, t\right)$ for every $n$ such that

$$
\int_{t_{n}+\tau-\sigma}^{\mu_{n}} \frac{q(s)}{r(s+\tau-\sigma)} d s \geq \frac{c}{2} \quad \text { and } \quad \int_{\mu_{n}}^{t_{n}} \frac{q(s)}{r(s+\tau-\sigma)} d s \geq \frac{c}{2} .
$$

Integrating both sides of (3.12) over the interval $\left[t_{n}, \mu_{n}\right]$ and $\left[\mu_{n}, t_{n}+\sigma-\tau\right]$, we have

$$
y\left(\mu_{n}\right)-y\left(t_{n}\right)+\frac{k_{0}\left(p_{0}-1\right)}{p_{0}^{2}} \int_{t_{n}}^{\mu_{n}} \frac{q(s)}{r(s+\tau-\sigma)} y(s+\tau-\sigma) d s=0
$$

and

$$
y\left(t_{n}+\sigma-\tau\right)-y\left(\mu_{n}\right)+\frac{k_{0}\left(p_{0}-1\right)}{p_{0}^{2}} \int_{\mu_{n}}^{t_{n}-\tau+\sigma} \frac{q(s)}{r(s+\tau-\sigma)} y(s+\tau-\sigma) d s=0 .
$$

From (3.19), (3.20), and (3.21), we have

$$
-y\left(t_{n}\right)+\frac{c}{2} y\left(\mu_{n}+\sigma-\tau\right) \leq 0
$$

and

$$
-y\left(\mu_{n}\right)+\frac{c}{2} y\left(t_{n}\right) \leq 0 .
$$

This implies eventually

$$
\frac{y\left(\mu_{n}-\sigma+\tau\right)}{y\left(\mu_{n}\right)} \leq\left(\frac{2}{c}\right)^{2}
$$

which is a contradiction with (3.18). The proof is complete.

Example 3.5 Consider the equation

$$
\left[e^{t}(x(t)+2 x(t-1))\right]^{\prime}+\frac{t+1}{t} e^{t-1} x(t-2)\left(1+x^{4}(t-2)\right)=0, \quad t \geq 2 .
$$

Here we have,

$$
\begin{aligned}
& r(t)=e^{t}, \quad a(t)=1, \quad q(t)=\frac{t+1}{t} e^{t-1}, \quad p_{0}=2, \quad \tau=1 \quad \text { and } \quad \sigma=2, \\
& f(x(t-\sigma))=x(t-2)\left(1+x^{4}(t-2)\right) .
\end{aligned}
$$

Then we have

$$
\left.\frac{f(u)}{u}=1+x^{4}(t-2)\right) \geq 1 .
$$

Let $k_{0}=1$; we have

$$
\begin{aligned}
\liminf _{t \rightarrow \infty} \int_{t}^{t-\tau+\sigma} \frac{q(s)}{r(s+\tau-\sigma)} d s & =\liminf _{t \rightarrow \infty} \int_{t}^{t+1}\left[\frac{e^{s-1}\left(\frac{s+1}{s}\right)}{e^{s-1}}\right] d s \\
& =\liminf _{t \rightarrow \infty} \int_{t}^{t+1}\left(1+\frac{1}{s}\right) d s \geq 1>0
\end{aligned}
$$


and

$$
\begin{aligned}
& \int_{t_{0}}^{\infty} \frac{q(t)}{r(t+\tau-\sigma)} \ln \left(\frac{e k_{0}\left(p_{0}-1\right)}{p_{0}^{2}} \int_{t}^{t+\sigma-\tau} \frac{q(s)}{r(s+\tau-\sigma)} d s\right) d t \\
& \quad=\int_{2}^{\infty}\left(\frac{t+1}{t} \ln \left(\frac{e}{4} \int_{t}^{t+1} \frac{s+1}{s} d s\right)\right) d t \geq \frac{e}{4} \int_{2}^{\infty} \ln \left(\ln \left(1+\frac{1}{t}\right)\right) d t=\infty .
\end{aligned}
$$

Hence, according to Theorem 3.1, all solutions of (3.22) oscillate.

Remark 3.6 Theorem 3.4 extends results of Graef et al. [10], Ahmed et al. [4], Saker and Elabbasy [21] and Saker and Kubiaczyk [8].

\section{Competing interests}

The authors declare that they have no competing interests.

\section{Authors' contributions}

This research has been done by FA under the general supervision of authors Prof. RA, Dr. UD, and Prof. MN, who conceived of the study, participated in its design and coordination and helped to draft the manuscript. All authors read and approved the final manuscript.

\section{Acknowledgements}

This research has been completed with the support of these grants: FRGS/2/2013/SG04/UKM/02/3 and DLP-2014-012.

Received: 30 September 2014 Accepted: 24 February 2015 Published online: 14 March 2015

\section{References}

1. Hale, JK: Theory of Functional Differential Equations. Springer, New York (1977)

2. Driver, RD: A mixed neutral system. Nonlinear Anal. 8, 155-158 (1984)

3. Boe, E, Chang, HC: Dynamics of delayed systems under feedback control. Chem. Eng. Sci. 44, 1281-1294 (1989)

4. Ahmed, FN, Ahmad, RR, Din, UKS, Noorani, MSM: Some further results on oscillations for neutral delay differential equations with variable coefficients. Abstr. Appl. Anal. 2014, Article ID 579023 (2014)

5. Karpuz, B, Ocalan, O: Oscillation criteria for some classes of linear delay differential equations of first order. Bull. Inst. Math. Acad. Sin. 3, 293-314 (2008)

6. Gopalsamy, K, Lalli, BS, Zhang, BG: Oscillation of odd order neutral differential equations. Czechoslov. Math. J. 42, 313-323 (1992)

7. Zhou, Y: Oscillation of neutral functional differential equations. Acta Math. Hung. 86, 205-212 (2000)

8. Saker, SH, Kubiaczyk, I: Oscillation of solutions to nonlinear neutral delay differential equations. J. Appl. Anal. 8, 261-278 (2002)

9. Kubiaczyk, I, Saker, SH, Morchalo, J: New oscillation criteria for first order nonlinear neutral delay differential equations. Appl. Math. Comput. 14, 225-242 (2003)

10. Graef, JR, Savithri, R, Thandapani, E: Oscillation of first order neutral delay differential equations. Electron. J. Qual. Theory Differ. Equ. Proc. 7'th Coll. Qualitative Theory of Diff. Equ. 2003, 12 (2003)

11. Agarwal, RP, Bohner, M, Li, WT: Nonoscillation and Oscillation: Theory for Functional Differential Equations, vol. 267. Dekker, New York (2004)

12. Ahmed, FN, Ahmad, RR, Din, UKS, Noorani, MSM: Oscillations for neutral functional differential equations. Sci. World J. 2014, Article ID 124310 (2014)

13. Ahmed, FN, Ahmad, RR, Din, UKS, Noorani, MSM: Oscillations for nonlinear neutral delay differential equations with variable coefficients. Abstr. Appl. Anal. 2014, Article ID 179195 (2014)

14. Chuanxi, Q, Ladas, G, Zhang, BG, Zhao, T: Sufficient conditions for oscillation and existence of positive solutions. Appl. Anal. 35, 187-194 (1990)

15. Dong, J-G: Oscillation of solutions for first order neutral differential equations with distributed deviating arguments. Comput. Math. Appl. 58, 784-790 (2009)

16. Elabbasy, EM, Hassan, TS, Saker, SH: Oscillation of nonlinear neutral delay differential equations. J. Appl. Math. Comput. 21, 99-118 (2006)

17. Jaros, J, Kusano, T: Oscillation properties for delay equations. Rocky Mt. J. Math. 29, 197-207 (1999)

18. Gyori, I, Ladas, G: Oscillation Theory of Delay Differential Equations with Applications. Clarendon, Oxford (1991)

19. Li, B: Oscillation of first order delay differential equations. Proc. Am. Math. Soc. 124, 3729-3737 (1996)

20. Erbe, LH, Kong, Q, Zhang, BG: Oscillation Theory for Functional Differential Equations. Dekker, New York (1995)

21. Saker, SH, Elabbasy, EM: Oscillation of first order neutral delay differential equations. Kyungpook Math. J. 41, 311-321 (2001) 